\title{
Actividad nocturna de Nyssomyia yuilli (Young \& Porter, 1972) y Nyssomyia trapidoi (Farchild \& Hertig, 1952) (Diptera: Psychodidae: Phlebotominae) en el municipio de Otanche (Boyacá, Colombia), zona endémica de leishmaniasis cutánea
}

Nocturnal activity of Nyssomyia yuilli (Young \& Porter, 1972) and Nyssomyia trapidoi (Farchild \& Hertig, 1952)

(Diptera: Psychodidae: Phlebotominae), in Otanche Municipality (Boyacá, Colombia), endemic area of cutaneous leishmaniasis

\author{
David-C Martínez-D ${ }^{1}$; Julián-L Ávila-J²; Fredy Molano-R ${ }^{1}$
}

Forma de citar: Martínez Dueñas D, Ávila J, Molano F. Actividad nocturna de Nyssomyia yuilli (Young \& Porter, 1972) y Nyssomyia trapidoi (Farchild \& Hertig, 1952) (Diptera: Psychodidae: Phlebotominae) en el municipio de Otanche (Boyacá, Colombia), zona endémica de leishmaniasis cutánea. Rev Univ Ind Santander Salud. 50(2): 106-114. doi: http://dx.doi.org/10.18273/revsal.v50n2-2018002 (c) (1)

\section{Resumen}

Introducción: Nyssomyia yuilli (Young \& Porter, 1972) y Nyssomyia trapidoi (Farchild \& Hertig, 1952) son vectores de Leishmania panamensis (Lainson y Shaw, 1972), causal de leishmaniasis cutánea en varios países de América como Brasil, Colombia, Ecuador y Panamá. Objetivo: Describir la actividad nocturna de N. yuilli y $N$. trapidoi en el municipio de Otanche, zona endémica de leishmaniasis cutánea. Metodología: Los especímenes fueron colectadas por medio de trampas CDC localizadas en el intra y peri-domicilio de viviendas que tenían antecedentes o casos activos de personas infectadas con leishmaniasis cutánea. Resultados: $N$. yuilli presentó una mayor actividad cerca de la media noche, mientras que $N$. trapidoi no mostró ningún incremento y mantuvo una actividad constante durante toda la noche. Conclusiones: Se observó que N. yuilli tiene una conducta típica de una especie de bosque con picos de actividad a media noche, mientras que $N$. trapidoi tiene un comportamiento de una especie antropofílica que se ha adaptado a la vida cerca de las viviendas humanas, con una actividad constante durante toda la noche sin ningún aumento esporádico.

Palabras clave: Diptera; Psychodidae; Vector; Conducta; Leishmaniasis cutánea; Colombia.

1. Universidad Pedagógica y Tecnológica de Colombia. Tunja, Colombia.

2. Secretaria Departamental de Salud - Gobernación de Boyacá. Tunja, Colombia.

Correspondencia: David Camilo Martínez Dueñas. Dirección: Calle 27A n.ํ 19-32 Tunja, Boyacá, Colombia. Teléfono: +57 3212211024. Correo electrónico: martínezd.camilo@gmail.com. 


\begin{abstract}
Introduction: Nyssomyia yuilli (Young \& Porter, 1972) and Nyssomyia trapidoi (Farchild \& Hertig, 1952) are vectors of Leishmania panamensis (Lainson y Shaw, 1972), causal of cutaneous leishmaniasis in several American countries such as Brazil, Colombia, Ecuador and Panama. Objective: Describe the nocturnal activity of N. yuilli and N. trapidoi in Otanche Municipality, endemic zone of cutaneous leishmaniasis. Methods: The specimens were collected through CDC traps located in the intra and peri-domicile of homes that had antecedents or active cases of infected people with cutaneous leishmaniasis. Results: N. yuilli present increased activity near midnight, while $N$. trapidoi not show any increase and maintained a constant activity throughout the night. Conclusions: It was observed that $N$. yuilli demonstrates typical conduct of a forest species, with peaks of activity at midnight, while $N$. trapidoi shows behaviour of an anthropophilic species that has been adapted to life near to human residences, with constant activity throughout the night without any sporadic increases.
\end{abstract}

Keywords: Diptera; Psychodidae; Vector; Behavior; Cutaneous leishmaniasis; Colombia.

\section{Introducción}

La leishmaniasis cutánea constituye un problema de salud pública debido a su morbilidad, amplia distribución geográfica y complejo ciclo de transmisión, comprendiendo diferentes especies de parásitos, reservorios y vectores ${ }^{1-3}$. A nivel mundial, más de 12 millones de personas están infectadas con leishmaniasis cutánea, y 350 millones están en riesgo de contraer esta enfermedad ${ }^{4}$. Para Colombia la situación es alarmante, ya que se presenta como uno de los países endémicos en América Latina ${ }^{5}$, segundo país con el mayor número de reportes positivos, solo superado por Brasil ${ }^{6}$.

Nyssomyia yuilli y Nyssomyia trapidoi (Psychodidae: Phlebotominae), son especies vectoras de flagelados del género Leishmania sp. (Euglenozoa: Trypanosomatidae), agente etiológico de la enfermedad tropical leishmaniasis , $^{4-8}$ en varios países de latinoamérica como Brasil, Colombia, Ecuador y Panamá9-14 . La especie $N$. trapidoi es considerada la principal especie transmisora de leishmaniasis cutánea en diferentes departamentos de Colombia, de los cuales, Nariño, Tolima, Antioquia, Boyacá y Santander, se le reconoce como un vector de Leishmania panamensis ${ }^{11,15-20}$; para este último, también se ha podido identificar a esta especie con individuos de L. (Viannia) braziliensis ${ }^{21}$. Para Colombia, se ha incriminado a $N$. yuilli como vector de L. panamensis encontrando hembras naturalmente infectadas en los municipios de Otanche y Pauna en el occidente del departamento de Boyacá $^{20}$, y para el municipio de Leticia (departamento del Amazonas) se encontraron individuos con flagelados no identificados ${ }^{9}$.

Así, debido a la presencia de $N$. yuilli y N. trapidoi en el municipio, y a los antecedentes vectoriales para el departamento y para el país ${ }^{20}$, se hace necesario el estudio de la bionomía de estas dos especies, investigaciones que ayuden a complementar y describir su ecología, en especial, parámetros de comportamiento como la actividad horaria de picadura $^{20,22}$, ya que la falta de información sobre los vectores agudiza la transmisión de la enfermedad, por la carencia de herramientas que faciliten la realización de planes de prevención y control $^{20,23-25}$. Por lo anterior, el objetivo de este estudio es describir la actividad nocturna de las especies de $N$. yuilli y $N$. trapidoi, en el municipio de Otanche, zona endémica de leishmaniasis cutánea.

\section{Metodología}

\section{Área de estudio}

El estudio se realizó en el municipio de Otanche, departamento de Boyacá, uno de los 25 municipios que más casos de leishmaniasis cutánea reportan a nivel nacional $(0,7$ del total de casos para el país a la semana epidemiológica 10 del 2014) ${ }^{21}$, y ubicado al occidente del departamento, área considerada como endémica para esta enfermedad ${ }^{20}$. Debido a que el mayor número de casos fueron reportados para el área rural $^{26}$, se seleccionó dentro del municipio la Vereda El Carmen (549'10,56”'N y 749'28,67'”, altura promedio de 915 $\mathrm{msnm}$ ) por el alto número de casos positivos (32 casos positivos a la semana epidemiológica 31 del 2014 $)^{27}$.

El área de estudio es un territorio propiamente montañoso y quebrado, presenta una temperatura media de $23-25^{\circ} \mathrm{C}$, con pisos térmicos cálido y templado, con zonas de vida como bosque húmedo tropical (bh-T), y bosque muy húmedo premontano (bmh-PM), con una pluviosidad anual entre $2800-3600 \mathrm{~mm}$ y un régimen de lluvias bimodal con máximos entre abril-mayo y octubre- noviembre ${ }^{28}$. 


\section{Recolección y determinación taxonómica}

La recolección de los flebótomos se llevó a cabo en el mes de agosto de 2014 durante 15 noches, donde se seleccionaron (de forma aleatoria) 41 viviendas con antecedentes de casos positivos de leishmaniasis cutánea o donde vivieran personas con ulceras activas, información adquirida por medio de encuestas entomológicas para estudios de foco de leishmaniasis cutánea $^{29-30}$. La colecta de flebótomos se realizó en tres viviendas por noche en promedio (dependiendo de las condiciones climáticas), siguiendo la metodología para levantamiento de adultos de flebótomos propuesta por el Instituto Nacional de Salud en la Guía: Protocolo para la Vigilancia en Salud Pública de leishmaniasis ${ }^{29}$.

En cada vivienda, previo a la consulta y firma de consentimiento informado, se instalaron dos trampas de luz blanca sin cebo, o trampas CDC (por sus siglas en inglés: Center for Disease Control and Prevention) por noche. En el intradomicilio (interior de la vivienda) en un dormitorio, y en el peridomicilio (parte exterior de la vivienda) en gallineros, establos o porquerizas, removiendo la malla cada hora durante doce horas (18:00-06:00). Se registraron datos ambientales como humedad relativa $(\%)$ y temperatura $\left({ }^{\circ} \mathrm{C}\right)$ promedio en cada hora de cada noche, con el objetivo de poder determinar si estos factores ambientales influyen sobre la actividad nocturna de los flebótomos.

El material recolectado en cada una de las trampas fue separado cuidadosamente, y los flebótomos encontrados se preservaron en alcohol al $70 \%$ para su posterior identificación en el Laboratorio de Entomología de la Secretaria de Salud del Departamento de Boyacá. La determinación de las especies se hizo por medio de la revisión de genitalitas de hembras y machos, con ayuda de las claves taxonómicas de Galati ${ }^{2}$ y Young y Duncan ${ }^{3}$, además, cada una de las especies fueron corroboradas por el programa de Control de Calidad del Instituto Nacional de Salud. El montaje permanente de los individuos se hizo en placas portaobjeto con bálsamo de Canadá31 y se incluyeron a la colección de referencia de Flebótomos de la Unidad de Entomología de Laboratorio Departamental de Salud Pública de Boyacá.

\section{Análisis de datos}

Para cada especie, incluyendo hembras y machos, se calculó la abundancia relativa por hora/por noche, lo que permitió determinar la actividad nocturna de las especies de flebótomos durante las 15 noches de muestreo. Para establecer si las variables ambientales temperatura y humedad están relacionadas o no con el número de individuos capturados, se utilizó una prueba paramétrica de correlación de Pearson, ya que esta indica la relación lineal entre variables ${ }^{32}$. Previo a esto, se realizó una prueba de Normalidad de Shapiro-Wilk. Todos los análisis se hicieron por medio del programa SPSS Statistics $18.1^{33}$.

\section{Resultados}

Se colectaron 361 individuos, distribuidos en 16 especies de la subfamilia Phlebotominae, de las cuales N. yuilli y $N$. trapidoi significaron el 60,3\% de todas las colectas. Para las dos especies el número de individuos capturados fue mayor en el intradomicilio (101) que en el peridomicilio (48), con más capturas de hembras que de machos, 102 y 47 individuos respectivamente. Tabla 1. Otras especies capturadas fueron Trichopygomyia triramula $(13,37 \%)$, Psathyromyia barrettoi majuscula (6,11\%), Lutzomyia hartmanni (4,17\%), Evandromyia dubitans (3,61\%), Ev. walkeri $(3,33 \%), L u$. sp. de pichinde (3,16\%), Ev. saulensis (1,94\%) y Lu. gomezi $(1,39 \%)$.

Tabla 1. Número de individuos (n) y abundancia relativa (\%) de N. yuilli y N. trapidoi, colectados en cada sitio de captura en la Vereda El Carmen, Otanche (Boyacá), durante 15 días del mes de agosto de 2014.

\begin{tabular}{|c|c|c|c|c|c|c|c|c|c|c|}
\hline \multirow{3}{*}{ Especie } & \multicolumn{4}{|c|}{ Intradomicilio } & \multicolumn{4}{|c|}{ Peridomicilio } & \multirow{2}{*}{\multicolumn{2}{|c|}{ Total }} \\
\hline & \multicolumn{2}{|r|}{$\delta$} & \multicolumn{2}{|c|}{ 우 } & \multicolumn{2}{|c|}{$\delta$} & \multicolumn{2}{|r|}{ 우 } & & \\
\hline & $\mathbf{n}$ & $(\%)$ & $\mathbf{n}$ & $(\%)$ & $\mathbf{n}$ & $(\%)$ & n & $(\%)$ & $\mathbf{n}$ & $(\%)$ \\
\hline Nyssomyia yuilli & 20 & $(13,42)$ & 51 & $(34,22)$ & 8 & $(5,36)$ & 15 & $(10,06)$ & 94 & $(63,08)$ \\
\hline Nyssomyia trapidoi & 9 & $(6,04)$ & 21 & $(14,09)$ & 10 & $(6,71)$ & 15 & $(10,06)$ & 55 & $(36,91)$ \\
\hline Total & 29 & & 72 & & 18 & & 30 & & 149 & \\
\hline
\end{tabular}

${ }^{a}$ Número de individuos colectados. 
La actividad de N. yuilli inició desde las 18:00 horas, alcanzando un incremento alrededor de la media noche entre las 23:00-24:00 horas, donde se capturó el 22\% de todos los individuos, a partir de esta hora empezó un descenso gradual hasta las 3:00-4:00 horas, donde se tuvieron las últimas colectas, a partir las 4:00 horas no se capturaron más individuos. Figura 1.

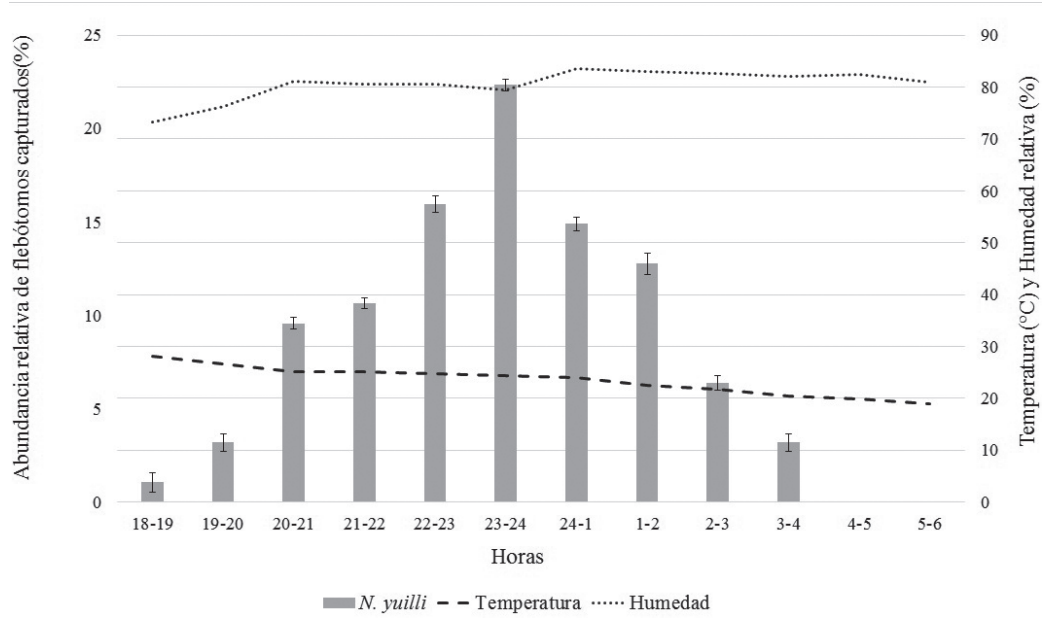

Figura 1. Actividad nocturna de N. yuilli. Se muestra la abundancia relativa de los individuos colectados por hora/por noche, desde las 18:00 hasta las 6:00 horas, durante 15 días del mes de agosto de 2014, en la Vereda El Carmen, Municipio Otanche (Boyacá).

$N$. trapidoi tuvo un inicio de actividad desde las 19:00 horas, aumentando sucesivamente hasta las 22:00 horas donde alcanzó un máximo, después de esta hora no se evidenciaron aumentos o disminuciones marcados hasta las 2:00-3:00 horas donde la actividad decae. A las 4:00-5:00 horas se registraron las últimas colectas para esta especie. Figura 2.

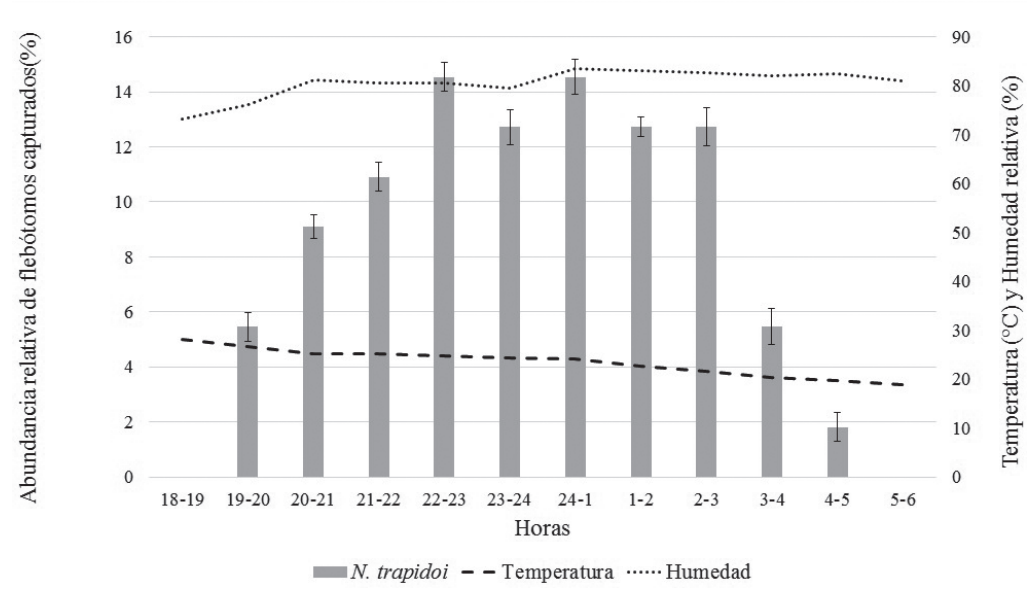

Figura 2. Actividad nocturna de $N$. trapidoi. Se muestra la abundancia relativa de los individuos colectados por hora/por noche, desde las 18:00 hasta las 6:00 horas, durante 15 días del mes de agosto de 2014, en la Vereda El Carmen, Municipio Otanche (Boyacá).

En cuanto a las condiciones meteorológicas durante las noches de muestreo, se presentó viento leve a moderado, lloviznas, cielo completamente o parcialmente cubierto, temperatura promedio de $23,5^{\circ} \mathrm{C}$ y humedad promedio de $80,5 \%$. Los resultados de los análisis de correlación de Pearson muestran que no existe una relación lineal entre la abundancia de los individuos colectados de la especie $N$. yuilli con la humedad relativa (r: 0,259, p: 0,416$)$ y temperatura promedio (r: 0,296, p: 0,351), igual resultado para la especie $N$. trapidoi: humedad relativa $(\mathrm{r}: 0,482, \mathrm{p}: 0,113)$ y temperatura promedio $(\mathrm{r}$ : 0,191, p: 0,552). 


\section{Discusión}

Para N. yuilli, Porter y De Foliart ${ }^{34}$ reportan un comportamiento con dos picos de actividad, en un bosque seco en el departamento de Antioquia (Colombia), el primero a las 20:00-21:00 horas y el segundo a las 24:00-1:00 horas, aparentando un comportamiento bimodal, puesto que los patrones de actividad fueron bastantes variables de una noche a otra, existiendo una sola constante: una mayor actividad a las primeras horas. Nuestros resultados difieren con lo anterior, ya que la alta actividad a horas tempranas de la noche no es alta, además, solo es evidente un aumento de actividad y no dos. Aun así, existe una similitud con el segundo incremento de actividad hacia la media noche reportado por Porter y De Foliart ${ }^{34}$. Este tipo de comportamiento, donde los flebótomos muestran una preferencia para alimentarse y ser más activas a mitad de la noche, constituye un comportamiento típico de especies de hábitat boscoso ${ }^{35-39}$, coincidiendo con el comportamiento de $L u$. intermedia y Lu. whitmani, especies típicas en selvas ecuatorianas ${ }^{40-41}$. Adicionalmente, nuestros resultados concuerdan con el criterio de Porter y De Foliart ${ }^{34}$ quienes describen a $N$. yuilli, como una especie característica de zonas boscosas, y con Young ${ }^{42}$, quien afirma que es una especie exclusiva de bosques en diferentes países de Suramérica. Sin embargo, como se observa en los resultados de este estudio y según López, et al..$^{43}$ y Alexander, et al. ${ }^{44}$, esta especie también está relacionada a ambientes modificados por el hombre, encontrándose en el intra y peridomicilio, lo cual confirma su adaptación a lugares donde la intervención humana está presente.

Con respecto a $N$. trapidoi, reportes para Colombia en un bosque seco tropical en el departamento de Antioquia ${ }^{34}$, describen una actividad desde las 6:00 horas, con un primer aumento a las 21:00-22:00 horas, y un segundo aumento a las 24:00-1:00 horas, manteniendo una actividad sin variaciones marcadas, comportamiento que se mantiene hasta las 3:00-4:00 horas donde la actividad empieza a decaer. En la vertiente del pacifico de los Andes en Ecuador, el comportamiento de esta especie difiere al de la población Colombiana, la cual exhibe un primer aumento de actividad a las 8:00-9:00 horas y un segundo con menor número de colectas a las 3:00-4:00 horas, a partir de la cual, el número de colectas empieza a disminuir ${ }^{12}$. El comportamiento de $N$. trapidoi en el área de estudio es similar con la población Colombiana y difiera en gran medida con la población en Ecuador, presentando una actividad que aumenta a medida que transcurre la noche con un máximo entre las
22:00-02:00 horas sin presentar cambios drásticos en la actividad, y con una disminución en la actividad a las 3:00-4:00 horas. Lo anterior evidencia que la actividad de esta especie no está limitada a una hora u horas específicas de la noche, comportamiento característico de una especie altamente antropofílica ${ }^{12,20,34}$, típica de flebótomos que están asociados a la presencia y actividad de los seres humanos, lo que sugiere una adaptación de esta especie a la conducta humana ${ }^{45}$.

En cuanto a la relación de la actividad nocturna con factores ambientales, estudios demuestran que las condiciones climáticas afectan la abundancia y presencia de los flebótomos adultos ${ }^{46-50}$, a pesar de esto, nuestros análisis describen que no existe una correlación positiva entre la actividad nocturna de $N$. yuilli y $N$. trapidoi con la temperatura y humedad, resultado que se asemeja a los expuestos por Porter y De Foliart ${ }^{34}$ y Hashiguchi ${ }^{12}$, donde la actividad de picadura de estas especies no tiene una relación con estos dos factores ambientales. De este modo, la baja pluviosidad registrada durante las capturas puede estar influyendo en el resultado, ya que en épocas lluvias, determinar una relación de la actividad de los flebótomos con la temperatura y la humedad es más difícil, pues a finales y después de estas, el número de capturas es mayor que durante la misma $^{50}$, como lo demuestran estudios sobre $L u$. evansi donde la mayor abundancia poblacional fue registrada después de los meses de mayor precipitación ${ }^{23,51-53}$. Otros factores que pueden estar incidiendo sobre la actividad de las dos especies son la velocidad del viento, el sexo de cada individuo, las diferencias en edades en la población y la atracción a la luz artificial de trampas y de las viviendas ${ }^{12,54-59}$. Este último, teniendo quizás una mayor incidencia, por lo que el alto número de capturas con relación a las demás especies, puede deberse a un fototropismo positivo hacia la luz artificial de las trampas CDC y por la cercanía de las viviendas a los bosques ${ }^{56,58,60}$.

Al ser las hembras las únicas hematófagas al picar a humanos y animales, son las responsables de la transmisión del agente etiológico ${ }^{61}$, por lo tanto, el alto número de hembras capturadas en comparación a los machos, puede tener una relación sobre la prevalencia de este enfermedad en la zona ${ }^{62}$, y más aún, si se tiene en cuenta que el número más alto de hembras capturado fue en el intradomicilio de las viviendas, situación documentada en varios estudios, donde los casos de leishmaniasis cutánea son frecuentes por el simple hecho de habitar cerca a los bosques ${ }^{63-66}$, lo que permite que la enfermedad se propague en ciclos extraforestales y aumente la prevalencia de esta en la zona ${ }^{67-69}$. 
Por último, la actividad nocturna de $N$. yuilli y N. trapidoi reportadas por Porter y De Foliart ${ }^{34}$, muestran que cada especie tiende a tener una pronunciada variación de una noche a otra, por lo que su actividad nocturna es muy fluctuante, incluso con patrones ambientales estables el comportamiento puede cambiar considerablemente, de manera que se hace necesario seguir estudiando los horarios de actividad de las especies de flebótomos para la zona teniendo en cuenta otras variables ambientales a las aquí estudiadas, abarcando diferentes épocas del año (época seca y época lluvia), e incluyendo áreas de bosque a parte del intra y peridomicilio.

\section{Agradecimientos}

Al laboratorio de la Secretaria de Salud de la Gobernación de Boyacá, en especial al grupo de Entomología y al grupo de Enfermedades Transmitidas por Vectores (ETV), por la colaboración en la parte logística durante las salidas de campo y la parte de laboratorio. A los técnicos de los municipios de Otanche y Pauna por su colaboración en la fase de campo. A la comunidad de la Vereda El Carmen del municipio de Otanche, por su amabilidad y disposición para los permisos de muestreos en las viviendas.

\section{Conflicto de intereses}

No se presentan ningún conflicto de interés por parte de los autores.

\section{Referencias}

1. Pan America Health Organization. Leishmaniasis: Epidemiological Report of the Americas. Report Leishmaniases $\mathrm{N}^{\circ} 1.2013$.

2. Galati EAB. Phlebotominae (Diptera, Psychodidae). Classificação, morfologia, terminologia e identificação de Adultos. 1 (ed). São Paulo: Universidade de São Paulo; 2013.

3. Young DG, Duncan MA. Guide to the identification and geographic distribution of Lutzomyia sand flies in Mexico, the West Indies, Central and South America (Diptera: Psychodidae). Florida: Memories of the American Entomological Institute; 1994.

4. Gradoni L, López-Vélez R, Mokni M. Manual on case management and surveillance of the leishmaniases in the WHO European Region. Copenhagen: World Health Organization; 2017.

5. Organización Panamericana de la Salud. Leishmaniasis: Informe Epidemiológico de las Américas. Informe de leishmaniasis No 4. 2016.
6. Organización Panamericana de la Salud. Leishmaniasis: Informe Epidemiológico de las Américas. Informe de leishmaniasis No 5. 2017.

7. Lainson R, Shaw JJ. New world leishmaniasis. In: Cox FEG, Wakelin D, Gillespie SH, Despommier D, editors. Parasitology. 10ed. London: Hodder Arnold; 2005: p.313-349.

8. Sharma U, Singh S. Insect vectors of Leishmania: distribution, physiology and their control. J Vector Borne Dis. 2008; 45(4): 255-272.

9. Arias JR, Miles MA, Naiff RD, Povoa MM, De Freitas RA, Bianciardi CB, et al. Flagellate infections of Brazilian sand flies (Diptera: Psychodidae): isolation in vitro and biochemical identification of Endotrypanum and Leishmania. Am J Trop Med Hyg. 1985; 34(6): 1098-1108.

10. Lainson R, Ryan L, Shaw JJ. Infective stages of Leishmania in the sand fly vector and some observations on the mechanism of transmission. Mem Inst Oswaldo Cruz. 1987; 82(3): 421-424.

11. Vélez ID, Ospina S, Henao G, Lepape P, Correa M, Wolff M, et al. Epidemiología de la leishmaniasis en San Roque, Antioquia. Boletín Epidemiológico de Antioquia. 1987; 12: 354-359.

12. Hashiguchi Y, Gomez EA, De Coronel VV, Mimori T, Kawabata M. Biting activity of two anthropophilic species of sandflies, Lutzomyia, in an endemic area of leishmaniasis in Ecuador. Ann Trop Med Parasitol. 1985; 79(5): 533-538.

13. Le Ponti F, Leon R, Guerrini F, Gantier JC, Mouchet $\mathrm{J}$, Echeverria R, et al. Leishmaniasis in Ecuador. 3. Lutzomyia trapidoi, vector of Leishmania panamensis. Ann Soc Belg Med Trop. 1994; 74(1): 23-28.

14. Christensen HA, Fairchild GB, Herre A, Johnson CM, Young DC, De Vasquez Am. The Ecology of cutaneous leishmaniasis in the Republic of Panama. J Med Entomol. 1983; 20(5): 463-484.

15. Morales A, Corredor A, Cáceres E, Ibagos AL, Rodríguez CI. Aislamiento de tres cepas de Leishmania a partir de Lutzomyia trapidoi en Colombia. Biomédica. 1981; 1(4): 37-41. doi: https://doi.org/10.7705/biomedica.v1i4.1802.

16. Young DG, Morales A, Kreutzer RD, Alexander JB, Corredor A, Tesh RB et al. Isolation of Leishmania braziliensis (Kinetoplastida: Trypanosomatidae) from cryopreserved Colombian sand flies (Diptera: Psychodidae). J Med Entomol. 1987; 5: 587-589.

17. Loyola EG, Álzate A, Sanchez A, González A. Epidemiology of a natural focus of Leishmania braziliensis in the Pacific lowlands of Colombia. III Natural infections in wilds mammals. Trans R Soc Trop Med Hyg. 1988; 82: 406-407.

18. Duque P, Vélez I, Morales M, Sierra D. Sand flies 
fauna involved in the transmission of cutaneous leishmaniasis in Afro-Colombian and American communities of Chocó, Pacific coast of Colombia. Neotrop Entomol. 2004; 33(2): 255-264. doi: http:// dx.doi.org/10.1590/S1519-566X2004000200018.

19. Molina JA. Determinación de la fauna flebotominea en el noroccidente de Boyacá. Implicación de Lutzomyia trapidoi como especie vectora en el foco de leishmaniasis de la Zambera (trabajo de grado). Bogotá: Universidad de los Andes; 1995.

20. Santamaría E, Ponce N, Zipa Y, Ferro C. Presencia en el peridomicilio de vectores infectados con Leishmania (Viannia) panamensis en dos focos endémicos en el occidente de Boyacá, piedemonte del valle del Magdalena medio, Colombia. Biomédica. 2006; 26(Supp 1): 82-94.

21. Muñoz G. Incriminación de vectores de Leishmania panamensis por métodos estadísticos. En: Memorias XXVI Congreso Sociedad Colombiana de Entomología. Santa Fe de Bogotá: Sociedad Colombiana de Entomología; 1999: p.164-171.

22. Pardo R, Farieta S, Munstermann L, Ferro C. Estudio preliminar de los flebótomos de Villeta y Quebradanegra Cundinamarca: sus implicaciones en salud pública. Biomédica. 1996; 16: 293-302.

23. Travi B, Montoya J, Gallego J, Jaramillo C, Llano R, Vélez I. Bionomics of Lutzomyia evansi (Diptera: Psychodidae) vector of visceral leishmaniasis in northern Columbia. J Med Entomol. 1996; 33(3): 278-285.

24. Davies C, Reithinger R, Campbell-Lendrum D, Feliciangeli D, Borges R, Rodriguez N. The Epidemiology of Leishmaniasis in Andean Countries. Cad Saúde Pública. 2000; 16(4): 925-950.

25. Feliciangeli MD. Hourly Activity of Lutzomyia ovallesi and L. gomezi (Diptera: Psychodidae), Vectors of Cutaneous Leishmaniasis in Northcentral Venezuela. J Med Entomol. 1997; 34(2): 110-115.

26. Instituto Nacional de Salud. Boletín Epidemiológico Semanal: Semana Epidemiológica No 10. 2014.

27. Sistema Nacional de Vigilancia en Salud Pública. Instituto Nacional de Salud, Secretaria Departamental de Salud, Gobernación de Boyacá. 2014.

28. Guerrero V. Plan Municipal de Desarrollo: Otanche vamos pa'lante, municipio de Otanche. Otanche: Departamento de Boyacá; 2013.

29. Instituto Nacional De Salud Pública. Guía: protocolo para la vigilancia en salud pública de Leishmaniasis. Instituto Nacional de Salud. 2006.

30. Zambrano P. Protocolo de vigilancia en salud pública. Leishmaniasis. 1 (ed). Santa Fé de Bogotá: Instituto Nacional de Salud, 2014.

31. Fuenzalida D. Técnica de clarificación y montaje para determinación de flebótomos. en: actas segunda reunión Red de Investigación de las Leishmaniasis en Argentina. Puerto Iguazú: Instituto Nacional de Medicina Tropical (INMeT); 2011: p. 34-37.

32. Guisande C, Vaamonde A, Barreiro A. Tratamiento de datos con R, STATISTICA y SPSS. 1 (ed). Vigo: Editorial Díaz de Santos; 2013.

33. IBM Corp. IBM SPSS Statistics para Windows. 18.1 version. Armonk: IBM Corp.; 2017.

34. Porter CH, De Foliart GR. The manbiting activity of phlebotomine sand flies (Diptera: Psychodidae) in a tropical wet forest environment in Colombia. Arq Zool S Paulo. 1981; 30: 81-158.

35. Williams P. The biting rhythms of some antropophilic Phlebotominae sand flies in British Honduras. Ann Trop Med Parasitol. 1966; 60: 357-364.

36. Disney RHL. Observations on a zoonosis: leishmaniasis in British Honduras. J Appl Ecol. 1986; 5: 1-59.

37. Morrison AMY, Ferro C, Pardo R, Torres M, Wilson ML, Tesh RB. Nocturnal activity patterns of Lutzomyia longipalpis (Diptera: Psychodidae) at an endemic focus of visceral leishmaniasis in Colombia. J Med Entomol. 1995; 32: 605-617.

38. Gonzalez R, Sousa L, Devera R, Jorquera A, Ledezma E. Seasonal and nocturnal domiciliary landing/ biting behaviour of Lutzomyia (Lutzomyia) evansi and Lutzomyia (Psychodopygus) panamensis (Diptera, Psychodidae) in a periurban area of city on the Caribbean coast of eastern Venezuela (Barcelona; Anzoategui State). Trans. R. Soc Trop Med Hyg. 1999; 93: 361-364.

39. Souza NA, Andrade CA, Peixoto AA, Rangel EF. Nocturnal activity rhythms of Lutzomyia intermedia and Lutzomyia whitmani (Diptera: Psychodidae) in a transmission area of American Cutaneous Leishmaniasis in Rio de Janeiro State, Brazil. J Med Entomol. 2005; 42(6): 986-992.

40. Teodoro U, Salvia FV, Lima EM, Spinosa RP, Barbosa OC, Ferreira MEM, et al. Flebotomõneos em area de transmissao de leishmaniose tegumentar na regiao norte do Estado do Parana-Brasil. Rev Sau de Publ. 1993; 27: 190-194. doi: http://dx.doi. org/10.1590/S0034-89101993000300006.

41. Falqueto A. Especificidade Alimentar de Flebotomíneos em duas áreas endêmicas de Leishmaniose Tegumentar no Estado do Espírito Santo (Tese de Doutorado). Rio de Janeiro: Instituto Oswaldo Cruz, Fundação Oswaldo Cruz, 1995: p. 84.

42. Young DG. A review of the bloodsucking psychodid flies of Colombia (Diptera: Phlebotominae and Sycoracinae). Gainesville: Universidad de la Florida; 1979. 
43. López Y, Osorio L, Álvarez G, Rojas J, Jiménez F, Gómez C, et al. Sandfly Lutzomyia longipalpis in a cutaneous leishmaniasis focus in Central Colombia. Mem Inst Oswaldo Cruz. 1999; 91: 415-419.

44. Alexander B, Agudelo LA, Navarro F, Ruiz F, Molina J, Aguilera G, et al. Phlebotomine sandflies and leishmaniasis risk in Colombian coffee plantations under two systems of cultivation. Med Vet Entomol. 2001; 15: 364-373.

45. Ferro C, Marin D, Góngora R, Carrasquilla MC, Trujillo JE, Rueda NK. Phlebotominae vector ecology in the domestic transmission of american cutaneous leishmaniasis in Chaparral, Colombia. J Med Entomol. 2011; 85(5): 847-856. doi: 10.4269/ ajtmh.2011.10-0560

46. Condino ML, Sampaio SM, Henriques LF, Galati EAB, Wanderley DM, Corrêa FM. Leishmaniose tegumentar americana: flebotomíneos de área de transmissão no município de Teodoro Sampaio, região sudoeste do Estado de São Paulo, Brasil. Rev Soc Brás Med Trop. 1998; 31(4): 355360. doi: http://dx.doi.org/10.1590/S003786821998000400004.

47. Salomón OD, Rossi GC, Cousiño B, Spinelli GR, Arias AR, Puerto DGL et al. Phlebotominae sandflies in Paraguay. Abundance distribution in the Southeastern region. Mem Inst Oswaldo Cruz. 2003; 98(2): 185-190. doi: http://dx.doi.org/10.1590/ S0074-02762003000200004.

48. Perruolo G. Aspectos ecológicos de Lutzomyia spp. (Diptera: Psychodidae) en un foco endémico de leishmaniasis cutánea en el Estado Táchira, Venezuela. Bol Malariol Salud Ambient. 2004; 44(1): 35-44.

49. Oliveira AG, Galati EAB, Fernandes CE, Dorval $\mathrm{ME}$, Peçanha R. Seasonal variation of Lutomyia longipalpis (Lut \& Neiva, 1912) (Diptera: Psychodidae: Phlebotominae) in endemic area of visceral leishmaniasis, Campo Grande, state of Mato Grosso do Sul, Brazil. Acta Trop. 2008; 105(1): 55-61.

50. Martínez-Suárez C, Almanza-Rodríguez C, Bejarano EE. Estimación del tiempo de desarrollo de Lutzomyia evansi bajo condiciones experimentales. Salud Uninorte. 2012; 28(1): 201-209.

51. Cruz LF, Manjarrez G, Bejarano EE. Variación temporal de especies de Lutzomyia (Diptera: Psychodidae) en el área urbana de Sincelejo (Colombia). Sal Uninorte. 2012; 28(2): 191-200.

52. Vélez ID, Travi B, Gallego J, Palma GI, Agudelo S, Montoya $\mathrm{J}$ et al. Evaluación ecoepidemiológica de la Leishmaniasis Visceral en la comunidad indígena zenú de San Andrés de Sotavento, Córdoba. Primer paso para su control. Rev Colomb Entomol. 1995; 21(3): 111-122.

53. Cochero S. Papel de Lutomyia evansi como vector de leishmaniasis visceral en Los Montes de María (Tesis de grado). Sincelejo: Universidad de Sucre; 2002.

54. Quate LW. Phlebotomus sandflies of the Paloich area in the Sudan. J Med Entomol. 1964; 1: 213-268.

55. Hoogstraal H, Dietlein DR, Heyneman D. Leishmanisis in the Sudan Republic. 4. Preliminary observations on man-biting sandflies (Psychodidae: Phlebotomus) in certain Upper Nile endemic areas. Trans R Soc Trop Med Hyg. 1962; 56: 411-422.

56. Martínez OE. Estudio taxonómico y faunístico de los flebótomos (Diptera, Psychodidae) del sureste español (Tesis de grado). Murcia: Sec. Biológicas; 1982.

57. Rubio-Palis Y, Curtis CF. Biting and resting behavior of anophelines in western Venezuela and implications for control of malaria transmission. Med Vet Entomol. 1992; 6: 325-334.

58. González CF. Estudio de los Phlebotomus spp. como factor de Leishmaniosis en el Somontano Oriental de la Provincia de Huesca. Lucas Mallada. 1994; 6: 101-129.

59. Rubio-Palis Y, Bevilacqua M, Medina DA, Moreno JE, Cárdenas L, Sánchez V, et al. Malaria entomological risk factors in relation to land cover in the Lower Caura River Basin, Venezuela. Mem Inst Oswaldo Cruz. 2013; 108: 220-228.

60. Chaniotis BN, Correa MA, Tesh RB, Johnson KM. Daily and season man-biting activity of Phlebotominae sandflies in Panama. J Med Entomol. 1971; 4: 415-420.

61. Lucientes J, Castillo J, Gracia M, Peribáñez M. 2005. Flebótomos, de la biología al control. Departamento de Patología Animal. Parasitología y Enfermedades Parasitarias. Redvet. 2005; 1(8): 1-8.

62. Desjeux P. Leishmaniasis: current situation and new perspectives. Comp Immunol Microbiol Infect Dis. 2004; 27: 305-318.

63. Andrade SL. Leishmaniose tegumentar americana em área de ocupação recente na periferia da cidade de Manaus Estado do Amazonas Brasil (Dissertação de Mestrado). Rio de Janeiro: Departamento de Medicina Tropical, Instituto Oswaldo Cruz; 1997.

64. Guerra JAO, Barros MLB, Guerra MVF, Talhari S, Paes MG. Leishmaniose tegumentar no Município de Manaus-aspectos epidemiológicos. Rev Soc Bras Med Trop. 1998; 31: 172.

65. Paes MG, Fé NF, Coelho LIARC, Oliveira RS, Sousa JU, Teixeira MR, et al. Sobre a ocorrência de leishmaniose tegumentar em rua de bairro de implantação antiga na Cidade de Manaus (AM). 
Rev Soc Bras Med Trop. 1998; 31: 75.

66. Talhari S, Arias JA, Cunha MGS, Naiff RD, Freitas RA, Barrett T. Leishmaniose no Estado do Amazonas-aspectos epidemiológicos clínicos e terapêuticos. An Bras Dermatol. 1988; 63: 433-438.

67. Dias ES, França-Silva JC, Silva JC, Monteiro EM, Paula KM, Gonçalves CM, et al. Flebotomíneos (Diptera: Psychodidae) de um foco de leishmaniose tegumentar no estado de Minas Gerais. Rev Soc Bras Med Trop. 2007; 40: 49-52.

68. Madeira MF, Uchoa CMA, Leal CA, Silva RM, Duarte R, Magalhães CM, et al. Leishmania (Viannia) braziliensis em cães naturalmente infectados. Rev Soc Bras Med Trop. 2003; 36: 551-555.

69. Monteiro EM, França-Silva JC, Costa RT, Costa DC, Barata RA, Paula EV, et al. Leishmaniose visceral: estudo de fl ebotomíneos e infecção canina em Montes Claros, Minas Gerais. Rev Soc Bras Med Trop. 2005; 38: 147-152. doi: http://dx.doi. org/10.1590/S0037-86822005000200004. 